International Journal of Pure and Applied Mathematics

Volume $95 \quad$ No. 2 2014, 137-147

ISSN: 1311-8080 (printed version); ISSN: 1314-3395 (on-line version)

url: http://www.ijpam.eu

doi: http://dx.doi.org/10.12732/ijpam.v95i2.3

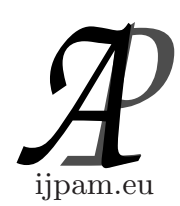

\title{
COEFFICIENT ESTIMATE OF CERTAIN SUBCLASSES \\ OF CONVEX $p$-VALENT FUNCTIONS WITH \\ A BOUNDED POSITIVE REAL PART
}

\author{
O.S. Babu ${ }^{1}$, C. Selvaraj ${ }^{2}$, G. Murugusundaramoorthy ${ }^{3}$ § \\ ${ }^{1}$ Department of Mathematics \\ Dr. Ambedkar Govt. Arts College \\ Chennai, 600039, INDIA \\ ${ }^{2}$ Department of Mathematics \\ Presidency College (Autonomous) \\ Chennai, 600005, INDIA \\ ${ }^{3}$ Corresponding Author \\ School of Advanced Sciences \\ VIT University \\ Vellore, 632014, INDIA
}

\begin{abstract}
We estimate the bounds of coefficients and solve Fekete-Szegö problem for $p$-valent Mocanu-convex and Pascu-type functions in the open unit disk $\triangle$ which maps $\triangle$ onto the strip domain $w$ with $p \alpha<\Re w<p \beta$.
\end{abstract}

AMS Subject Classification: 30C45, 30C50

Key Words: analytic function, univalent function, $p$ - valent function, starlike function, Mocanu-convex function, Pascu-type function, subordination, coefficient estimate, Fekete-Szegö problem

Received: February 23, 2014

(C) 2014 Academic Publications, Ltd. url: www.acadpubl.eu

$\S_{\text {Correspondence author }}$ 


\section{Introduction}

Let $\mathcal{A}_{p}$ denote the class of all functions $f(z)$ of the form

$$
f(z)=z^{p}+\sum_{n=1}^{\infty} a_{p+n} z^{p+n} \quad(p \in \mathbb{N}=\{1,2,3, \ldots\})
$$

which are analytic and $p$-valent in the open unit disk $\triangle=\{z: z \in \mathbb{C}:|z|<1\}$. Note that $\mathcal{A}_{1}:=\mathcal{A}$ the class of analytic functions further $\mathcal{S}$ the subclass of $\mathcal{A}$ consisting of all univalent functions $f$ in $\triangle$. A function $f \in \mathcal{A}$ is said to be starlike of order $\alpha(0 \leq \alpha<1)$ in $\triangle$ if it satisfies $\Re\left(\frac{z f^{\prime}(z)}{f(z)}\right)>\alpha$. This class is denoted by $\mathcal{S}^{*}(\alpha)$ and $\mathcal{S}^{*}(0)=\mathcal{S}^{*}$. The class $\mathcal{S}^{*}(\alpha)$ was introduced by Robertson [4]. It is well-known that $\mathcal{S}^{*}(\alpha) \subset \mathcal{S}^{*} \subset \mathcal{S}$. Furthermore, let $\mathcal{M}(\beta)$ be the class of functions $f \in \mathcal{A}$ which satisfy $\Re\left(\frac{z f^{\prime}(z)}{f(z)}\right)<\beta \quad(z \in \triangle)$ for some real number $\beta$ with $\beta>1$. The class $\mathcal{M}(\beta)$ was investigated by Uralegaddi et. al $[6]$.

Let $P(z)$ and $Q(z)$ be analytic in $\triangle$. Then the function $P(z)$ is said to subordinate to $Q(z)$ in $\triangle$ written by

$$
P(z) \prec Q(z) \quad(z \in \triangle),
$$

if there exists a function $w(z)$ which is analytic in $\triangle$ with $w(0)=0$ and $|w(z)|<$ $1(z \in \triangle)$, and such that $P(z)=Q(w(z))(z \in \triangle)$. From the definition of the subordinations, it is easy to show that the subordination (1) implies that

$$
P(0)=Q(0) \quad \text { and } \quad P(\triangle) \subset Q(\triangle) .
$$

In particular, if $Q(z)$ is univalent in $\triangle$, then the subordination (1) is equivalent to the condition (2).

Motivated by the classes $\mathcal{S}^{*}(\alpha)$ and $\mathcal{M}(\beta)$, we define a new class for certain $p$-valent functions.

Definition 1. Let $\alpha$ and $\beta$ be real numbers such that $0 \leq \alpha<1<\beta$. The function $f \in \mathcal{A}_{p}$ belongs to the class $\mathcal{S}^{p}(\alpha, \beta)$ if $f$ satisfies the following inequality

$$
\alpha<\Re\left(\frac{z f^{\prime}(z)}{p f(z)}\right)<\beta \quad(z \in \triangle, p \in \mathbb{N}) .
$$

Definition 2. Let $\alpha$ and $\beta$ be real numbers such that $0 \leq \alpha<1<\beta$. The function $f \in \mathcal{A}_{p}$ belongs to the class $\mathcal{C}_{\lambda}^{p}(\alpha, \beta)$ if $f$ satisfies the following inequality

$$
\alpha<\Re\left\{\frac{1}{p}\left(1+\frac{z f^{\prime \prime}(z)}{f^{\prime}(z)}\right)\right\}<\beta \quad(z \in \triangle, \lambda \geq 0) .
$$


Definition 3. Let $\alpha$ and $\beta$ be real numbers such that $0 \leq \alpha<1<\beta$. The function $f \in \mathcal{A}_{p}$ belongs to the class $\mathcal{M}_{\lambda}^{p}(\alpha, \beta)$ if $f$ satisfies the following inequality

$$
\alpha<\Re\left\{\frac{(1-\lambda)}{p} \frac{z f^{\prime}(z)}{f(z)}+\frac{\lambda}{p}\left(1+\frac{z f^{\prime \prime}(z)}{f^{\prime}(z)}\right)\right\}<\beta \quad(z \in \triangle, \lambda \geq 0, p \in \mathbb{N}) .
$$

Remark 4. When $p=1, \mathcal{M}_{\lambda}^{p}(\alpha, \beta)$ reduces to $\mathcal{M}_{\lambda}(\alpha, \beta)$, the class of Mocanu-convex functions with bounded positive real part. Further we note that $\mathcal{M}_{0}^{p}(\alpha, \beta)=\mathcal{S}^{p}(\alpha, \beta)[1], \mathcal{M}_{0}^{1}(\alpha, \beta)=\mathcal{S}(\alpha, \beta)[3], \mathcal{M}_{1}^{p}(\alpha, \beta)=\mathcal{C}^{p}(\alpha, \beta)$, the class of $p$-valent convex functions with bounded positive real part and $\mathcal{M}_{1}^{1}(\alpha, \beta)=\mathcal{C}(\alpha, \beta)$, the class of convex functions with bounded positive real part.

Definition 5. Let $\alpha$ and $\beta$ be real numbers such that $0 \leq \alpha<1<\beta$. The function $f \in \mathcal{A}_{p}$ belongs to the class $\mathcal{N}_{\lambda}^{p}(\alpha, \beta)$ if $f$ satisfies the following inequality

$$
\alpha<\Re\left\{\frac{(1-\lambda) \frac{1}{p} z f^{\prime}(z)+\lambda \frac{1}{p^{2}} z\left(z f^{\prime}(z)\right)^{\prime}}{(1-\lambda) f(z)+\lambda \frac{1}{p} z f^{\prime}(z)}\right\}<\beta \quad(z \in \triangle, \lambda \geq 0, p \in \mathbb{N}) .
$$

Remark 6. When $p=1, \mathcal{N}_{\lambda}^{p}(\alpha, \beta)$ reduces to $\mathcal{N}_{\lambda}(\alpha, \beta)$, the class of Pascu-type functions with positive real part. Further we note that $\mathcal{N}_{0}^{p}(\alpha, \beta)=$ $\mathcal{S}^{p}(\alpha, \beta)[1], \mathcal{N}_{0}^{1}(\alpha, \beta)=\mathcal{S}(\alpha, \beta)[3], \mathcal{N}_{1}^{p}(\alpha, \beta)=\mathcal{C}^{p}(\alpha, \beta)$ and $\mathcal{N}_{1}^{1}(\alpha, \beta)=$ $\mathcal{C}(\alpha, \beta)$.

Now, we define an analytic function $\mathcal{S}_{\alpha, \beta}(z): \triangle \longrightarrow \mathbb{C}$ by

$$
\mathcal{S}_{\alpha, \beta}(z)=1+\frac{\beta-\alpha}{\pi} i \log \left(\frac{1-e^{\frac{i \pi(1-\alpha)}{\beta-\alpha} z}}{1-e^{-\frac{i \pi(1-\alpha)}{\beta-\alpha}} z}\right)
$$

due to Kuroki and Owa [3] and they proved $\mathcal{S}_{\alpha, \beta}(z)$ maps $\triangle$ onto a convex domain $w$ with $\alpha<\Re(w)<\beta$, conformaly.

\section{Coefficient Estimates for $f \in \mathcal{M}_{\lambda}^{p}(\alpha, \beta)$}

Applying the function $\mathcal{S}_{\alpha, \beta}(z)$ defined by (7), we give a necessary and sufficient condition for $f(z) \in \mathcal{A}_{p}$ to belong to the class $\mathcal{M}_{\lambda}^{p}(\alpha, \beta)$. 
Lemma 7. Let $f(z) \in \mathcal{A}_{p}$ and $0 \leq \alpha<1<\beta$. Then $f(z) \in \mathcal{M}_{\lambda}^{p}(\alpha, \beta)$ if and only if

$$
\frac{(1-\lambda)}{p} \frac{z f^{\prime}(z)}{f(z)}+\frac{\lambda}{p}\left(1+\frac{z f^{\prime \prime}(z)}{f^{\prime}(z)}\right) \prec 1+\frac{\beta-\alpha}{\pi} i \log \left(\frac{1-e^{i \frac{\pi(1-\alpha)}{\beta-\alpha} z}}{1-e^{-i \frac{\pi(1-\alpha)}{\beta-\alpha}} z}\right)
$$

in $\triangle$.

By taking $\lambda=0$ and $\lambda=1$ we state the following lemmas respectively:

Lemma 8. [1] Let $f \in \mathcal{A}_{p}$. Then $f(z) \in \mathcal{S}^{p}(\alpha, \beta)$ if and only if

$$
\frac{z f^{\prime}(z)}{p f(z)} \prec 1+\frac{\beta-\alpha}{\pi} i \log \left(\frac{1-e^{i \frac{\pi(1-\alpha)}{\beta-\alpha} z}}{1-e^{-i \frac{\pi(1-\alpha)}{\beta-\alpha}} z}\right) \quad(z \in \triangle)
$$

where $\alpha<1$ and $\beta>1$.

Lemma 9. Let $f \in \mathcal{A}_{p}$. Then $f(z) \in \mathcal{C}^{p}(\alpha, \beta)$ if and only if

$$
\frac{1}{p}\left(1+\frac{z f^{\prime \prime}(z)}{f^{\prime}(z)}\right) \prec 1+\frac{\beta-\alpha}{\pi} i \log \left(\frac{1-e^{i \frac{\pi(1-\alpha)}{\beta-\alpha} z}}{1-e^{-i \frac{\pi(1-\alpha)}{\beta-\alpha} z}}\right) \quad(z \in \triangle)
$$

where $\alpha<1$ and $\beta>1$.

We note that

$$
\mathcal{S}_{\alpha, \beta}(z)=1+\frac{\beta-\alpha}{\pi} i \log \left(\frac{1-e^{i \frac{\pi(1-\alpha)}{\beta-\alpha} z}}{1-e^{-i \frac{\pi(1-\alpha)}{\beta-\alpha}} z}\right)=1+\sum_{n=1}^{\infty} B_{n} z^{n},
$$

where

$$
B_{n}=\frac{2(\beta-\alpha)}{n \pi} \sin \frac{n \pi(1-\alpha)}{\beta-\alpha} \quad(n=1,2,3, \ldots) .
$$

Using the subordination (8), we find sharp bounds on the second and third coefficients for $f(z) \in \mathcal{M}_{\lambda}^{p}(\alpha, \beta)$, by applying the following lemma due to Rogosinki $[5]$.

Lemma 10. Let $P(z)=\sum_{n=1}^{\infty} A_{n} z^{n}$ and $Q(z)=\sum_{n=1}^{\infty} B_{n} z^{n}$ be analytic in $\triangle$. If $P(z) \prec Q(z)(z \in \triangle)$, then

$$
\sum_{k=1}^{m}\left|A_{k}\right|^{2} \leq \sum_{k=1}^{m}\left|B_{k}\right|^{2} \quad(m=1,2, \ldots) .
$$


Theorem 11. If the function $f(z)=z^{p}+\sum_{n=1}^{\infty} a_{p+n} z^{p+n} \in \mathcal{M}_{\lambda}^{p}(\alpha, \beta)$, then

$$
\left|a_{p+1}\right| \leq \frac{2 p^{2}(\beta-\alpha)}{\pi(p+\lambda)} \sin \frac{\pi(1-\alpha)}{\beta-\alpha}
$$

and

$$
\begin{aligned}
& \left|a_{p+2}\right| \leq \frac{2 p^{2}(\beta-\alpha)}{\pi(p+\lambda)} \sin \frac{\pi(1-\alpha)}{\beta-\alpha} \\
& \quad \times\left(\cos \frac{\pi(1-\alpha)}{\beta-\alpha}+\frac{2 p\left(p^{2}+2 p \lambda+\lambda\right)(\beta-\alpha)}{\pi(p+\lambda)^{2}} \sin \frac{\pi(1-\alpha)}{\beta-\alpha}\right) .
\end{aligned}
$$

Proof. Let

$$
\begin{aligned}
P(z) & =\frac{(1-\lambda)}{p} \frac{z f^{\prime}(z)}{f(z)}+\frac{\lambda}{p}\left(1+\frac{z f^{\prime \prime}(z)}{f^{\prime}(z)}\right) \\
& =1+\frac{(1+p \lambda)}{p} a_{p+1} z+\left[\frac{2(p+2 \lambda)}{p^{2}} a_{p+2}-\frac{\left(p^{2}+2 p \lambda+\lambda\right)}{p^{3}} a_{p+1}^{2}\right] z^{2}+\cdots
\end{aligned}
$$

and

$$
Q(z)=\mathcal{S}_{\alpha, \beta}(z)=1+\sum_{n=1}^{\infty} B_{n} z^{n},
$$

where $B_{n}$ is as in (11). Applying Lemma 10 we can get the results as asserted.

When $\lambda=0$ and $\lambda=1$ we state the following corollaries respectively:

Corollary 12. [1] If the function $f(z)=z^{p}+\sum_{n=1}^{\infty} a_{p+n} z^{p+n} \in \mathcal{S}^{p}(\alpha, \beta)$, then

$$
\left|a_{p+1}\right| \leq \frac{2 p(\beta-\alpha)}{\pi} \sin \frac{\pi(1-\alpha)}{\beta-\alpha}
$$

and

$$
\left|a_{p+2}\right| \leq \frac{p(\beta-\alpha)}{\pi} \sin \frac{\pi(1-\alpha)}{\beta-\alpha}\left(\cos \frac{\pi(1-\alpha)}{\beta-\alpha}+\frac{2 p(\beta-\alpha)}{\pi} \sin \frac{\pi(1-\alpha)}{\beta-\alpha}\right) .
$$

Corollary 13. If the function $f(z)=z^{p}+\sum_{n=1}^{\infty} a_{p+n} z^{p+n} \in \mathcal{C}^{p}(\alpha, \beta)$, then

$$
\left|a_{p+1}\right| \leq \frac{2 p^{2}(\beta-\alpha)}{\pi(p+1)} \sin \frac{\pi(1-\alpha)}{\beta-\alpha}
$$


and

$$
\left|a_{p+2}\right| \leq \frac{p^{2}(\beta-\alpha)}{\pi(p+2)} \sin \frac{\pi(1-\alpha)}{\beta-\alpha}\left[\cos \frac{\pi(1-\alpha)}{\beta-\alpha}+\frac{2 p(\beta-\alpha)}{\pi} \sin \frac{\pi(1-\alpha)}{\beta-\alpha}\right] .
$$

When $p=1$, from Theorem 11, we state the following corollary:

Corollary 14. If the function $f(z)=z+\sum_{n=2}^{\infty} a_{n} z^{n} \in \mathcal{M}_{\lambda}(\alpha, \beta)$, then

$$
\left|a_{2}\right| \leq \frac{2(\beta-\alpha)}{\pi(1+\lambda)} \sin \frac{\pi(1-\alpha)}{\beta-\alpha}
$$

and

$$
\left|a_{3}\right| \leq \frac{(\beta-\alpha)}{\pi(1+2 \lambda)} \sin \frac{\pi(1-\alpha)}{\beta-\alpha}\left(\cos \frac{\pi(1-\alpha)}{\beta-\alpha}+\frac{2(1+3 \lambda)(\beta-\alpha)}{\pi(1+\lambda)^{2}} \sin \frac{\pi(1-\alpha)}{\beta-\alpha}\right) .
$$

Making use of the following lemma we shall solve the Fekete-Szegö problem for $f(z) \in \mathcal{M}_{\lambda}^{p}(\alpha, \beta)$.

Lemma 15. [2]Let $h(z)=1+h_{1} z+h_{2} z^{2}+\cdots$ be a function with positive real part in $\triangle$. Then for any complex number $\nu$,

$$
\left|h_{2}-\nu h_{1}^{2}\right| \leq 2 \max \{1,|1-2 \nu|\} .
$$

Theorem 16. Let $0 \leq \alpha<1<\beta$ and let the function $f$ given by $f(z)=z^{p}+\sum_{n=1}^{\infty} a_{p+n} z^{p+n}$ be in the class $\mathcal{M}_{\lambda}^{p}(\alpha, \beta)$. Then for any complex number $\mu$,

$$
\begin{aligned}
& \left|a_{p+2}-\mu a_{p+1}^{2}\right| \leq \frac{p^{2}(\beta-\alpha)}{\pi(p+2 \lambda)} \sin \frac{\pi(1-\alpha)}{\beta-\alpha} \\
\times & \max \left\{1,\left|\cos \frac{\pi(1-\alpha)}{\beta-\alpha}+\frac{2 p\left[\left(p^{2}+2 p \lambda+\lambda\right)-2 \mu p(p+2 \lambda)\right](\beta-\alpha)}{\pi(p+\lambda)^{2}} \sin \frac{\pi(1-\alpha)}{\beta-\alpha}\right|\right\} .
\end{aligned}
$$

Proof. Let $P(z)=\frac{(1-\lambda)}{p} \frac{z f^{\prime}(z)}{f(z)}+\frac{\lambda}{p}\left(1+\frac{z f^{\prime \prime}(z)}{f^{\prime}(z)}\right)$. Then, since $f \in$ $\mathcal{M}_{\lambda}^{p}(\alpha, \beta)$, we have $P(z) \prec Q(z)$, where $Q(z)$ is given by (12).

Let

$$
h(z)=\frac{1+Q^{-1}(P(z))}{1-Q^{-1}(P(z))}=1+h_{1} z+h_{2} z^{2}+\cdots \quad(z \in \triangle) .
$$

Then $h$ is analytic and has positive real part in the open disk $\triangle$. We also have

$$
P(z)=Q\left(\frac{h(z)-1}{h(z)+1}\right) \quad(z \in \triangle) .
$$


We find from the equation (13) that

$$
\begin{aligned}
& a_{p+1}=\frac{p^{2} B_{1} h_{1}}{(p+\lambda)} \\
& a_{p+2}=\frac{p^{2}}{2(p+2 \lambda)}\left[\frac{B_{2} h_{1}^{2}}{4}-\frac{B_{1} h_{1}^{2}}{4}+\frac{B_{1} h_{2}}{2}+\frac{p\left(p^{2}+2 p \lambda+\lambda\right)}{(p+\lambda)^{2}} B_{1}^{2} h_{1}^{2}\right]
\end{aligned}
$$

which imply that

$$
a_{p+2}-\mu a_{p+1}^{2}=\frac{p^{2} B_{1}}{4(p+2 \lambda)}\left(h_{2}-\nu h_{1}^{2}\right)
$$

where

$$
\nu=\frac{1}{2}\left(1-\frac{B_{2}}{B_{1}}-\frac{p\left(p^{2}+2 p \lambda+\lambda\right) B_{1}}{(p+\lambda)^{2}}+\frac{2 \mu p^{2}(p+2 \lambda) B_{1}}{(p+\lambda)^{2}}\right) .
$$

Applying Lemma 15, we obtain

$$
\begin{aligned}
\left|a_{p+2}-\mu a_{p+1}^{2}\right| & =\frac{p^{2}}{4(p+2 \lambda)}\left|B_{1}\right|\left|h_{2}-\nu h_{1}^{2}\right| \\
& \leq \frac{p^{2}}{2(p+2 \lambda)} B_{1} \max \{1,|1-2 \nu|\} .
\end{aligned}
$$

Substituting $B_{1}=\frac{2(\beta-\alpha)}{\pi} \sin \frac{\pi(1-\alpha)}{\beta-\alpha}$ and $B_{2}=\frac{(\beta-\alpha)}{\pi} \sin \frac{2 \pi(1-\alpha)}{\beta-\alpha}$ in (14), we can obtain the results as asserted.

By taking $\lambda=0$ and $\lambda=1$ we state the following corollaries respectively:

Corollary 17. [1] Let $0 \leq \alpha<1<\beta$ and let the function $f$ given by $f(z)=z^{p}+\sum_{n=1}^{\infty} a_{p+n} z^{p+n}$ be in the class $\mathcal{S}^{p}(\alpha, \beta)$. Then for any complex number $\mu$,

$$
\begin{aligned}
\left|a_{p+2}-\mu a_{p+1}^{2}\right| & \leq \frac{p(\beta-\alpha)}{\pi} \sin \frac{\pi(1-\alpha)}{\beta-\alpha} \\
& \times \max \left\{1,\left|\cos \frac{\pi(1-\alpha)}{\beta-\alpha}+\frac{2 p(1-2 \mu)(\beta-\alpha)}{\pi} \sin \frac{\pi(1-\alpha)}{\beta-\alpha}\right|\right\} .
\end{aligned}
$$

Corollary 18. Let $0 \leq \alpha<1<\beta$ and let the function $f$ given by $f(z)=z^{p}+\sum_{n=1}^{\infty} a_{p+n} z^{p+n}$ be in the class $\mathcal{C}^{p}(\alpha, \beta)$. Then for any complex number $\mu$, 


$$
\begin{aligned}
&\left|a_{p+2}-\mu a_{p+1}^{2}\right| \leq \frac{p^{2}(\beta-\alpha)}{\pi(p+2)} \sin \frac{\pi(1-\alpha)}{\beta-\alpha} \\
& \times \max \left\{1,\left|\cos \frac{\pi(1-\alpha)}{\beta-\alpha}+\frac{2 p\left[(p+1)^{2}-2 \mu p(p+2)\right](\beta-\alpha)}{\pi(p+1)^{2}} \sin \frac{\pi(1-\alpha)}{\beta-\alpha}\right|\right\} .
\end{aligned}
$$

Putting $p=1$ in Theorem 16, we get the following corollary.

Corollary 19. Let $0 \leq \alpha<1<\beta$ and let the function $f$ given by $f(z)=z+\sum_{n=2}^{\infty} a_{n} z^{n}$ be in the class $\mathcal{M}_{\lambda}(\alpha, \beta)$. Then for any complex number $\mu$,

$$
\begin{aligned}
& \left|a_{3}-\mu a_{2}^{2}\right| \leq \frac{(\beta-\alpha)}{\pi(1+2 \lambda)} \sin \frac{\pi(1-\alpha)}{\beta-\alpha} \\
& \times \max \left\{1,\left|\cos \frac{\pi(1-\alpha)}{\beta-\alpha}+\frac{2[(1+3 \lambda)-2 \mu(1+2 \lambda)](\beta-\alpha)}{\pi(1+\lambda)^{2}} \sin \frac{\pi(1-\alpha)}{\beta-\alpha}\right|\right\} .
\end{aligned}
$$

\section{Coefficient Estimates for $f \in \mathcal{N}_{\lambda}^{p}(\alpha, \beta)$}

First, by applying the function $\mathcal{S}_{\alpha, \beta}(z)$ defined by $(7)$, we give a necessary and sufficient condition for $f(z) \in \mathcal{A}_{p}$ to belong to the class $\mathcal{N}_{\lambda}^{p}(\alpha, \beta)$.

Lemma 20. Let $f(z) \in \mathcal{A}_{p}$ and $0 \leq \alpha<1<\beta$. Then $f(z) \in \mathcal{N}_{\lambda}^{p}(\alpha, \beta)$ if and only if

$$
\frac{(1-\lambda) \frac{1}{p} z f^{\prime}(z)+\lambda \frac{1}{p^{2}} z\left(z f^{\prime}(z)\right)^{\prime}}{(1-\lambda) f(z)+\lambda \frac{1}{p} z f^{\prime}(z)} \prec 1+\frac{\beta-\alpha}{\pi} i \log \left(\frac{1-e^{i \frac{\pi(1-\alpha)}{\beta-\alpha}} z}{1-e^{-i \frac{\pi(1-\alpha)}{\beta-\alpha}} z}\right)
$$

in $\triangle$.

Remark 21. For $\lambda=0$ and $\lambda=1$, we get Lemma 8 and Lemma 9 respectively.

Using the subordination (15), we find sharp bounds on the second and third coefficients for $f(z) \in \mathcal{N}_{\lambda}^{p}(\alpha, \beta)$, by applying Lemma 10 .

Theorem 22. If the function $f(z)=z^{p}+\sum_{n=1}^{\infty} a_{p+n} z^{p+n} \in \mathcal{N}_{\lambda}^{p}(\alpha, \beta)$, then

$$
\left|a_{p+1}\right| \leq \frac{2 p^{2}(\beta-\alpha)}{\pi(p+\lambda)} \sin \frac{\pi(1-\alpha)}{\beta-\alpha}
$$


and

$$
\left|a_{p+2}\right| \leq \frac{p^{2}(\beta-\alpha)}{\pi(p+2 \lambda)} \sin \frac{\pi(1-\alpha)}{\beta-\alpha}\left(\cos \frac{\pi(1-\alpha)}{\beta-\alpha}+\frac{2 p(\beta-\alpha)}{\pi} \sin \frac{\pi(1-\alpha)}{\beta-\alpha}\right) .
$$

Proof. Let

$$
\begin{aligned}
P(z) & =\frac{(1-\lambda) \frac{1}{p} z f^{\prime}(z)+\lambda \frac{1}{p^{2}} z\left(z f^{\prime}(z)\right)^{\prime}}{(1-\lambda) f(z)+\lambda \frac{1}{p} z f^{\prime}(z)} \\
& =1+\frac{(p+\lambda)}{p} a_{p+1} z+\left[\frac{2(p+2 \lambda)}{p^{2}} a_{p+2}-\frac{(p+\lambda)^{2}}{p^{3}} a_{p+1}^{2}\right] z^{2}+\cdots
\end{aligned}
$$

and

$$
Q(z)=\mathcal{S}_{\alpha, \beta}(z)=1+\sum_{n=1}^{\infty} B_{n} z^{n}
$$

where $B_{n}$ is as in (11). Applying Lemma 10 we can get the results as asserted.

Remark 23. For $\lambda=0$ and $\lambda=1$, we get Corollary 12 and Corollary 13 respectively.

When $p=1$, from Theorem 22, we state the following corollary:

Corollary 24. If the function $f(z)=z+\sum_{n=2}^{\infty} a_{n} z^{n} \in \mathcal{N}_{\lambda}(\alpha, \beta)$, then

$$
\left|a_{2}\right| \leq \frac{2(\beta-\alpha)}{\pi(1+\lambda)} \sin \frac{\pi(1-\alpha)}{\beta-\alpha}
$$

and

$$
\left|a_{3}\right| \leq \frac{(\beta-\alpha)}{\pi(1+2 \lambda)} \sin \frac{\pi(1-\alpha)}{\beta-\alpha}\left(\cos \frac{\pi(1-\alpha)}{\beta-\alpha}+\frac{2(\beta-\lambda)}{\pi} \sin \frac{\pi(1-\alpha)}{\beta-\alpha}\right) .
$$

Again using Lemma 15 we shall solve the Fekete-Szegö problem for $f(z) \in$ $\mathcal{N}_{\lambda}^{p}(\alpha, \beta)$.

Theorem 25. Let $0 \leq \alpha<1<\beta$ and let the function $f$ given by $f(z)=z^{p}+\sum_{n=1}^{\infty} a_{p+n} z^{p+n}$ be in the class $\mathcal{N}_{\lambda}^{p}(\alpha, \beta)$. Then for any complex number $\mu$,

$$
\begin{aligned}
&\left|a_{p+2}-\mu a_{p+1}^{2}\right| \leq \frac{p^{2}(\beta-\alpha)}{\pi(p+2 \lambda)} \sin \frac{\pi(1-\alpha)}{\beta-\alpha} \\
& \times \max \left\{1,\left|\cos \frac{\pi(1-\alpha)}{\beta-\alpha}+\frac{2 p\left[(p+\lambda)^{2}-2 \mu p(p+2 \lambda)\right](\beta-\alpha)}{\pi(p+\lambda)^{2}} \sin \frac{\pi(1-\alpha)}{\beta-\alpha}\right|\right\} .
\end{aligned}
$$


Proof. Let

$$
P(z)=\frac{(1-\lambda) \frac{1}{p} z f^{\prime}(z)+\lambda \frac{1}{p^{2}} z\left(z f^{\prime}(z)\right)^{\prime}}{(1-\lambda) f(z)+\lambda \frac{1}{p} z f^{\prime}(z)} .
$$

Then, since $f \in \mathcal{N}_{\lambda}^{p}(\alpha, \beta)$, we have $P(z) \prec Q(z)$, where $Q(z)$ is given by $(17)$.

Let

$$
h(z)=\frac{1+Q^{-1}(P(z))}{1-Q^{-1}(P(z))}=1+h_{1} z+h_{2} z^{2}+\cdots \quad(z \in \triangle) .
$$

Then $h$ is analytic and has positive real part in the open disk $\triangle$. We also have

$$
P(z)=Q\left(\frac{h(z)-1}{h(z)+1}\right) \quad(z \in \triangle) .
$$

We find from the equation (18) that

$$
\begin{aligned}
a_{p+1} & =\frac{p^{2} B_{1} h_{1}}{2(p+\lambda)} \\
a_{p+2} & =\frac{p^{2}}{2(p+2 \lambda)}\left[\frac{B_{2} h_{1}^{2}}{4}-\frac{B_{1} h_{1}^{2}}{4}+\frac{B_{1} h_{2}}{2}+\frac{p B_{1}^{2} h_{1}^{2}}{4}\right]
\end{aligned}
$$

which imply that

$$
a_{p+2}-\mu a_{p+1}^{2}=\frac{p^{2} B_{1}}{4(p+2 \lambda)}\left(h_{2}-\nu h_{1}^{2}\right)
$$

where

$$
\nu=\frac{1}{2}\left(1-\frac{B_{2}}{B_{1}}-\frac{p\left[(p+\lambda)^{2}-2 \mu p(p+2 \lambda)\right] B_{1}}{(p+\lambda)^{2}}\right) .
$$

Applying Lemma 15, we obtain

$$
\begin{aligned}
\left|a_{p+2}-\mu a_{p+1}^{2}\right| & =\frac{p^{2}}{4(p+2 \lambda)}\left|B_{1}\right|\left|h_{2}-\nu h_{1}^{2}\right| \\
& \leq \frac{p^{2}}{2(p+2 \lambda)} B_{1} \max \{1,|1-2 \nu|\}
\end{aligned}
$$

Substituting $B_{1}=\frac{2(\beta-\alpha)}{\pi} \sin \frac{\pi(1-\alpha)}{\beta-\alpha}$ and $B_{2}=\frac{(\beta-\alpha)}{\pi} \sin \frac{2 \pi(1-\alpha)}{\beta-\alpha}$ in (19), we can obtain the results as asserted.

Remark 26. By taking $\lambda=0$ and $\lambda=1$ we get Corollary 17 and Corollary 18 respectively. 
Putting $p=1$ in Theorem 25, we get the following corollary.

Corollary 27. Let $0 \leq \alpha<1<\beta$ and let the function $f$ given by $f(z)=z+\sum_{n=2}^{\infty} a_{n} z^{n}$ be in the class $\mathcal{N}_{\lambda}(\alpha, \beta)$. Then for any complex number $\mu$,

$$
\begin{aligned}
& \left|a_{3}-\mu a_{2}^{2}\right| \leq \frac{(\beta-\alpha)}{\pi(1+2 \lambda)} \sin \frac{\pi(1-\alpha)}{\beta-\alpha} \\
& \times \max \left\{1,\left|\cos \frac{\pi(1-\alpha)}{\beta-\alpha}+\frac{2\left[(1+\lambda)^{2}-2 \mu(1+2 \lambda)\right](\beta-\alpha)}{\pi(1+\lambda)^{2}} \sin \frac{\pi(1-\alpha)}{\beta-\alpha}\right|\right\} .
\end{aligned}
$$

Remark 28. By taking $\lambda=0$ and $\lambda=1$ one can deduce the results for functions $f \in \mathcal{S}_{\lambda}^{p}(\alpha, \beta)$ [1] and $f \in \mathcal{C}^{p}(\alpha, \beta)$ respectively. In particular $\lambda=0, p=1$ leads to the results obtained in [3].

\section{References}

[1] O.S. Babu, C. Selvaraj, G. Murugusundaramoorthy and S.Logu, Coefficient estimate of certain subclasses of convex $p$-valent Functions with a Bounded Positive Real Part (communicated).

[2] F. Keogh and E. Merkers, A coefficient inequality for certain classes of analytic functions, Proc. Amer. Math. Soc., 20 (1969), 8-12.

[3] K. Kuroki and S. Owa, Notes on new class for certain analytic functions, Advances in Mathematice: Scientific. Journal 1 (2012), no. 2, 127-131.

[4] M.S. Robertson, On the theory of univalent functions, Ann. Math. 37 (1936), 374-408.

[5] W. Rogosinski, On the coefficients of subordinate functions, Proc. London Math. Soc., 48 (1943), 48-82.

[6] B.A. Uralegaddi, M.D. Ganigi and S.M. Sarangi, Univalent functions with positive coefficients, Tamkang J. Math. 25 (1994), 225-230. 
